\title{
Aging with Traumatic Brain Injury: Effects of Age at Injury on Behavioral Outcome following Diffuse Brain Injury in Rats
}

\author{
Rachel K. Rowe ${ }^{a-c}$ Jenna M. Ziebella, b, e Jordan L. Harrison ${ }^{a, b}$, d \\ L. Matthew Law ${ }^{a, b}$ P. David Adelsona, b, d Jonathan Lifshitz ${ }^{a-d}$ \\ ${ }^{a}$ Barrow Neurological Institute at Phoenix Children's Hospital, ${ }^{b}$ Department of Child Health, University of \\ Arizona College of Medicine - Phoenix, and 'Phoenix Veteran Affairs Healthcare System, Phoenix, Ariz., and \\ dInterdisciplinary Graduate Program in Neuroscience, Arizona State University, Tempe, Ariz., USA; ${ }^{\text {eWicking }}$ \\ Dementia Research and Education Centre, Faculty of Health, University of Tasmania, Hobart, Tas., Australia
}

\section{Key Words}

Traumatic brain injury - Concussion - Aging · Puberty .

Behavior · Juvenile $\cdot$ Age at injury

\begin{abstract}
Development and aging are influenced by external factors with the potential to impact health throughout the life span. Traumatic brain injury (TBI) can initiate and sustain a lifetime of physical and mental health symptoms. Over 1.7 million TBIs occur annually in the USA alone, with epidemiology suggesting a higher incidence for young age groups. Additionally, increasing life spans mean more years to age with TBI. While there is ongoing research of experimental pediatric and adult $\mathrm{TBI}$, few studies to date have incorporated animal models of pediatric, adolescent, and adult TBI to understand the role of age at injury across the life span. Here, we explore repeated behavioral performance between rats exposed to diffuse TBI at five different ages. Our aim was to follow neurological morbidities across the rodent life span with respect to age at injury. A single cohort of male Sprague-
\end{abstract}

Dawley rats $(n=69)$ was received at postnatal day (PND) 10 . Subgroups of this cohort ( $n=11-12 /$ group) were subjected to a single moderate midline fluid percussion injury at age PND 17, PND 35, 2 months, 4 months, or 6 months. A control group of naïve rats $(n=12)$ was assembled from this cohort. The entire cohort was assessed for motor function by beam walk at 1.5, 3, 5, and 7 months of age. Anxiety-like behavior was assessed with the open field test at 8 months of age. Cognitive performance was assessed using the novel object location task at 8, 9, and 10 months of age. Depression-like behavior was assessed using the forced swim test at 10 months of age. Age at injury and time since injury differentially influenced motor, cognitive, and affective behavioral outcomes. Motor and cognitive deficits occurred in rats injured at earlier developmental time points, but not in rats injured in adulthood. In contrast, rats injured during adulthood showed increased anxiety-like behavior compared to uninjured control rats. A single diffuse TBI did not result in chronic depression-like behaviors or changes in body weight among any groups. The interplay of age at injury and aging with an injury are translationally important factors that influ-

\section{KARGER}

E-Mail karger@karger.com

www.karger.com/dne
(C) 2016 S. Karger AG, Basel

0378-5866/16/0383-0195\$39.50/0
Jonathan Lifshitz, PhD

Translational Neurotrauma Research Program

Barrow Neurological Institute at Phoenix Children's Hospital

1919 E. Thomas Road, Phoenix, AZ 85016 (USA)

E-Mail jlifshitz@email.arizona.edu 
ence behavioral performance as a quality of life metric. More complete understanding of these factors can direct rehabilitative efforts and personalized medicine for TBI survivors.

(C) 2016 S. Karger AG, Basel

\section{Introduction}

Over 1.7 million traumatic brain injuries (TBIs) occur annually in the USA, with incidence spread across all age groups [1]. TBI is more than a singular event, such that a disease state ensues with morbidities developing and persisting over an extended time frame following the initial insult. These chronic consequences are particularly profound for pediatric TBI, where decades of decreased quality of life can ensue [for review, see 2]. While experimental animal models of juvenile TBI have been developed, rarely have they been directly compared with models of adult TBI. The current study seeks to fill this gap in knowledge by assessing how age at the time of brain injury (age at injury) affects behavioral performance in multiple neurological domains over an extended time course.

Clinical evidence supports the persistence of neurological dysfunction following pediatric TBI, including cognitive and psychosocial impairments [for review, see 3]. Childhood is a time of rapid brain development, and it was previously assumed that this greater plasticity allowed for an increased ability to recover from injury, referred to as the early plasticity theory $[4,5]$. Although this theory has been supported by focal injury studies $[6,7]$, it does not fully explain recovery patterns following diffuse TBI $[6,8]$. Prevailing hypotheses suggest that TBI could disrupt ongoing development processes such as dendritization, synaptogenesis, and myelination, resulting in exacerbated morbidities across multiple neurological domains. TBI sustained during developmental childhood stages could lead to acute and chronic dysfunction across motor, cognitive, and psychological domains.

Beyond pediatrics, the adult population has the benefit of completing development, but also has ongoing cellular senescence. These ongoing life events can interact with the development of the TBI disease state as determined by an impact on neurological function $[9,10]$. At this stage, adult and old rats may have the experience, but not the capacity, to deal with injury [11]. Furthermore, the prospect of needing neurological function dwindles as injury occurs later in life. A recent review of clinical data suggests that elderly patients ( $<60$ years) are underrepresented in TBI research studies, and more research needs to be conducted to establish treatment end points for this aging population [12]. Although we agree the elderly represent a population vulnerable to TBI, we did not include this age group in the current study. Further studies are necessary to investigate the effects of TBI on the elderly population using experimental brain injury in aged animals ( $>10$ months).

Age at injury is likely to influence the way the brain is able to repair itself as a result of developmental status, extent of cellular senescence, and injury-induced inflammation $[6,13-15]$. The objective of the current study was to investigate the functional outcome from diffuse TBI in rodents brain-injured at different points throughout their life span. The novel study design addressed age at injury in an otherwise uniform cohort of rodents. We found that both age at time of injury and time since injury influenced somatic, cognitive, and affective behavioral outcomes. Both motor and cognitive deficits occurred in rats injured at earlier developmental time points, but not in rats injured in adulthood. In contrast, rats injured during adulthood showed increased anxiety-like behavior compared to uninjured control rats. A single diffuse TBI did not result in chronic depression-like behaviors or changes in body weight among any groups. Here we found that age at injury can be prescriptive of some, but not all, injuryinduced deficits.

\section{Experimental Procedures}

\section{Animals}

Adult male Sprague-Dawley rats (Harlan Laboratories, Inc., Indianapolis, Ind., USA) were used for all experiments. The rats were housed in a reversed light cycle with a 12-hour dark/light cycle (9:00/21:00 $\mathrm{h})$ at a constant temperature $\left(23 \pm 2{ }^{\circ} \mathrm{C}\right)$ with food and water available ad libitum according to the Association for Assessment and Accreditation of Laboratory Animal Care International. The rats were shipped at postnatal day (PND) 10 with the dam. They were acclimated to their environment following shipment for at least 7 days prior to any experiments. The rats were weaned from the dam at PND 21 and housed in pairs. After surgery, the rats were evaluated daily for 3 days by a physical examination, and documentation of each animal's condition was recorded. The Institutional Animal Care and Use Committees (IACUC) at the University of Arizona (Phoenix, Ariz., USA) approved the animal care.

\section{Synopsis of Experimental Procedures}

The following experiments used one cohort of rats. All pups $(\mathrm{n}=81)$ were shipped at PND 10 with the dam in a single shipment and assigned to treatment groups upon arrival. This cohort was used to assess time after injury and age at injury following midline fluid percussion injury (FPI), as previously described [16]. Groups were injured at PND 17, PND 35, 2 months, 4 months, or 6 months. At the time of weaning, the rats were housed in pairs with a litter mate from a different treatment group, such that no two cage 


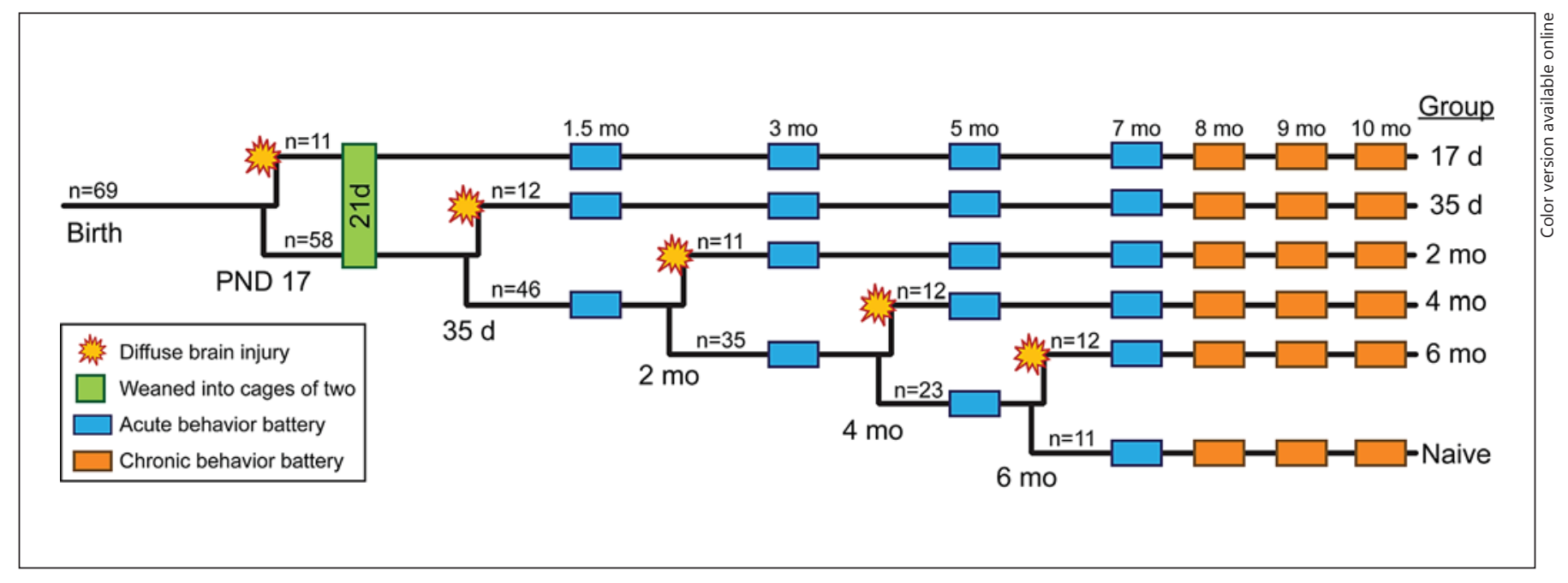

Fig. 1. Study design. The following experiments used one cohort of rats. All pups $(n=82)$ were shipped at PND 10 with the dam in a single shipment and assigned to treatment groups upon arrival. This cohort $(n=69)$ was used to assess time after injury and age at injury following midline fluid percussion injury. Groups were in- jured at PND 17, PND 35, 2 months, 4 months, or 6 months. Vestibulomotor function (acute behavior battery) was assessed at 1.5, 3,5 , and 7 months of age. Cognition, anxiety, and depressive-like behaviors were assessed at 8,9 , and 10 months of age. mates were injured at the same time. Behavior was assessed at 1.5, 3,5 , and 7-10 months of age (fig. 1). All animal studies were conducted in accordance with the guidelines established by the internal IACUC and the NIH guidelines for the care and use of laboratory animals. Studies are reported following the ARRIVE (Animal Research: Reporting in vivo Experiments) guidelines. Randomization of animals was achieved by assigning animals to treatment groups before the initiation of the study to ensure equal distribution across groups. A power analysis was performed to calculate group sizes to allow robust detection of injury-induced deficits while minimizing the number of animals required. This calculation was based on preliminary data and previously published work from our research group. Data collection stopped at the predetermined final end point of 10 months of age for each animal. Twelve rats were excluded from the study completely (this is not reflected in fig. 1). The reasons for exclusion were as follows: (1) 1 rat developed an epithelial tumor; (2) 1 rat aspirated following the forced swim task; (3) 1 rat was incorrectly sexed in the initial arrival screening and was removed along with its cage mate; (4) 2 rats (6 months old) were removed following injury because postinjury weight loss exceeded 15\% of presurgical weight; (5) 1 rat (35 days old) died following injury, and (6) 5 rats were excluded as surgical failures. Behavior data sets were screened using the extreme studentized deviate method for significant outliers, which are reported in Results. All animal behavior was scored by investigators blinded to the injury time point of the animal.

\section{Midline Fluid Percussion Injury}

The rats were subjected to midline FPI consistent with methods previously described [16-21]. The rats were anesthetized using 5\% isoflurane in $100 \%$ oxygen for $5 \mathrm{~min}$, and the head of the rat was placed in a stereotaxic frame with continuously delivered isoflurane at $2.5 \%$ via nosecone. While anesthetized, body temperature was maintained using a Deltaphase ${ }^{\circledR}$ isothermal heating pad (Braintree Scientific Inc., Braintree, Mass., USA). A midline incision was made exposing bregma and lambda, and fascia was removed from the surface of the skull. Trephines of varying outer diameters - $3.0 \mathrm{~mm}$ (PND 17), $4.0 \mathrm{~mm}$ (PND 35), and $4.8 \mathrm{~mm}$ (2, 4 , and 6 months) - were used for the craniotomy, centered on the sagittal suture between bregma and lambda without disruption of the dura. An injury cap prepared from the female portion of a Luer-Lok needle hub was fixed over the craniotomy using cyanoacrylate gel and methyl-methacrylate (Hygenic Corp., Akron, Okla., USA). The incision was sutured at the anterior and posterior edges, and topical Lidocaine ointment was applied. The rats were placed in a heated recovery cage and monitored until ambulatory.

For injury induction, the rats were reanesthetized (60-90 min after surgery) with $5 \%$ isoflurane delivered for $5 \mathrm{~min}$. The dura was visually inspected through the hub to make sure it was intact with no debris. The hub was then filled with normal saline and attached to the male end of the fluid percussion device (Custom Design and Fabrication, Virginia Commonwealth University, Richmond, Va., USA). The PND 17 rats were connected to the device using a LuerLok extension tube. An injury of moderate severity for this injury model was administered by releasing the pendulum onto the fluidfilled cylinder. To achieve moderate severity the pressure was adjusted across age groups based on age and body weight -1.5 atm (PND 17), 1.9 atm (PND 35), 2.1 atm (2 months), 2.2 atm (4 months), and 2.7 atm (6 months). The rats were monitored for the presence of seizures, apnea, and righting reflex times (table 1). Seizures were defined as abnormal posturing or spontaneous movement during the period of righting reflex suppression. Apnea was recorded when breathing ceased for greater than $5 \mathrm{~s}$ immediately following injury, as previous published [22]. The righting reflex time is the total time from the initial impact until the rat spontane- 


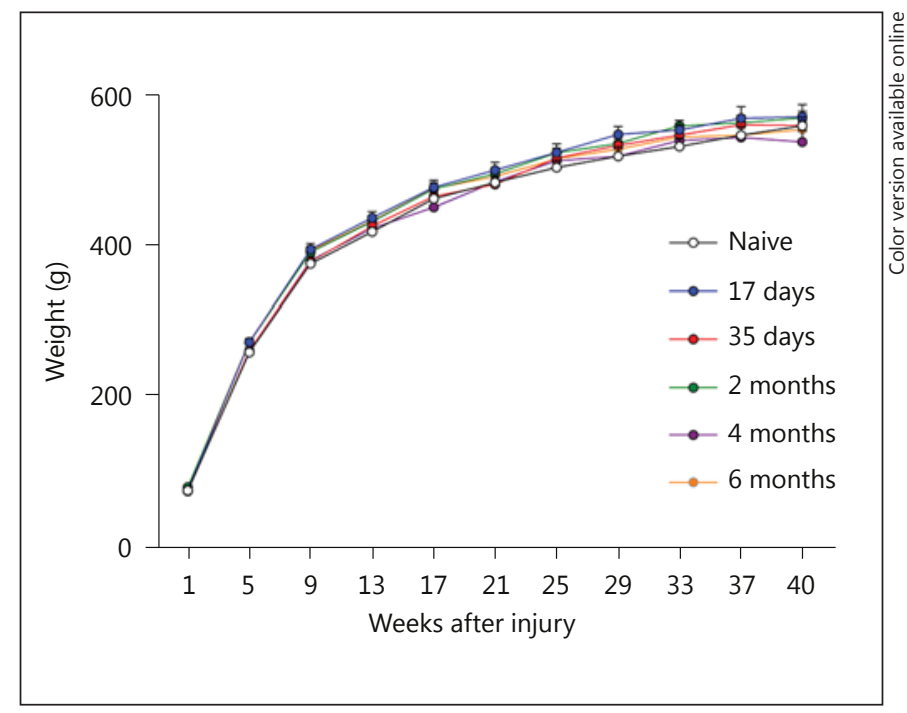

Fig. 2. Age at injury did not influence body weight. Beginning 1 week after injury, rats were weighed every 4 weeks for the duration of the study. Though all groups gained weight over time, no significant effect of age at injury was detected between groups. Data are presented as mean body weight \pm SEM; naïve: $\mathrm{n}=12$, 17 days: $\mathrm{n}=11,35$ days: $\mathrm{n}=13,2$ months: $\mathrm{n}=14,4$ months: $\mathrm{n}=16,6$ months: $\mathrm{n}=16$.

Table 1. Physiological responses to diffuse brain injury as a function of age at injury

\begin{tabular}{lllll}
\hline & $\begin{array}{l}\text { Terminal } \\
\text { weight, }\end{array}$ & $\begin{array}{l}\text { Seizure } \\
\text { presence, } \\
\mathrm{n}\end{array}$ & $\begin{array}{l}\text { Apnea } \\
\text { presence, } \\
\mathrm{n}\end{array}$ & Apnea, s \\
& & NA & $\mathrm{NA}$ & $\mathrm{NA}$ \\
\hline Naïve & $552.80 \pm 11.93$ & $\mathrm{NA}$ & \\
17 days & $577.27 \pm 12.95$ & $7 / 11$ & $11 / 11$ & $40.09 \pm 11.53$ \\
35 days & $560.67 \pm 17.34$ & $4 / 12$ & $3 / 12$ & $13.33 \pm 2.85$ \\
2 months & $569.00 \pm 11.12$ & $0 / 11$ & $0 / 11$ & 0 \\
4 months & $536.75 \pm 17.64$ & $1 / 12$ & $1 / 12$ & 11.00 \\
6 months & $552.83 \pm 12.61$ & $1 / 12$ & $0 / 12$ & 0 \\
\hline
\end{tabular}

No significant differences in terminal weight were detected $\left(F_{5,63}=0.9847, p=0.4342\right)$. Injury-induced seizures and apnea were prevalent in juvenile rats, with over half the rats injured at PND 17 having seizure and apnea. Seizures were defined as abnormal posturing or spontaneous movement during the period of righting reflex suppression. Apnea was recorded when breathing ceased for more than $5 \mathrm{~s}$ immediately following injury. NA = Not applicable.

ously rights itself from a supine position. A righting reflex time of 5-10 min for all age groups was indicative of moderate injury and used as inclusion criteria for the study, as previously published [19, 23-26]. In juvenile rats (PND 17), the presence of a seizure shortened the righting reflex times as juveniles consistently righted themselves immediately following injury-induced seizure. After the injury, the hub was removed and the brain was inspected for uniform herniation and integrity of the dura. The dura was intact in all rats; none were excluded as technical failures. The incision was cleaned using saline and closed using sutures. After spontaneously righting, the rats were placed in a heated recovery cage and monitored until ambulatory (approx. 5-15 $\mathrm{min}$ ) before being returned to their cage. Adequate measures were taken to minimize pain or discomfort.

\section{Postoperative Weight}

The weight of each rat was recorded on the day of surgery. Following surgery, the animals were postoperatively monitored for the first 3 days after injury. This monitoring included a physical examination, examination of the suture site, and evaluation of changes in postoperative weight. The rats were weighed biweekly for 10 months, and a terminal weight was recorded (table 1). Body weight did not differ between groups receiving diffuse TBI at different ages (fig. 2).

\section{Behavioral Testing}

All behavioral testing occurred on the same day for the entire cohort. Behavior was tested under red lighting between 09:00 and 16:00 h. All behavior was performed by individuals blinded to the animals' treatment groups.

\section{Beam Walk}

A beam walk task was used to evaluate vestibulomotor function and coordination. The rats were trained 2 consecutive days prior to the day of testing. The rats were trained using negative reinforcement to escape white noise by traversing an elevated narrow beam $(3 \times 100 \mathrm{~cm})$ and entering a dark goal box placed on the opposite end $[27,28]$. The beam walk was assessed by recording the time to traverse the beam and the foot faults that occurred while traversing the beam. Each rat performed two trials; time and foot faults were averaged. If a rat turned around on the beam and walked in the wrong direction, the trial was excluded.

\section{Open Field}

The open field task was used to assess locomotor activity and anxiety-like behavior, as previously described [29]. At 8 months after injury, the rats were placed in an empty arena $(70 \times 70 \times 46$ $\mathrm{cm}, \mathrm{W} \times \mathrm{L} \times \mathrm{H}$ ) and allowed to explore freely for $5 \mathrm{~min}$. Their movement was tracked by an overhead camera and the distance traveled, time spent in the center of the arena $(42 \times 42 \mathrm{~cm})$, and number of entries into the center were calculated using EthoVision XT 10 software (Noldus Information Technology, Leesburg, Va., USA).

\section{Novel Object Location Task}

Cognitive impairment was tested using the novel location of object task, as previously published [30]. The test consisted of three phases: habituation, training, and testing. At 8, 9, and 10 months after injury, the rats were placed in an open field $(70 \times 70 \times 46 \mathrm{~cm}$, $\mathrm{L} \times \mathrm{W} \times \mathrm{H}$ ) for $5 \mathrm{~min}$ of habituation. The rats were removed and two identical objects were placed in opposing quadrants of the field for the training phase. The rats were placed in the center of the open field and given 3 min to explore the objects. Following the training, they were returned to their home cages for $3 \mathrm{~min}$ and one object was relocated to an adjacent quadrant (objects and arena were not cleaned). Following the object relocation, the rats were placed back into the center of the arena for the testing phase. The 


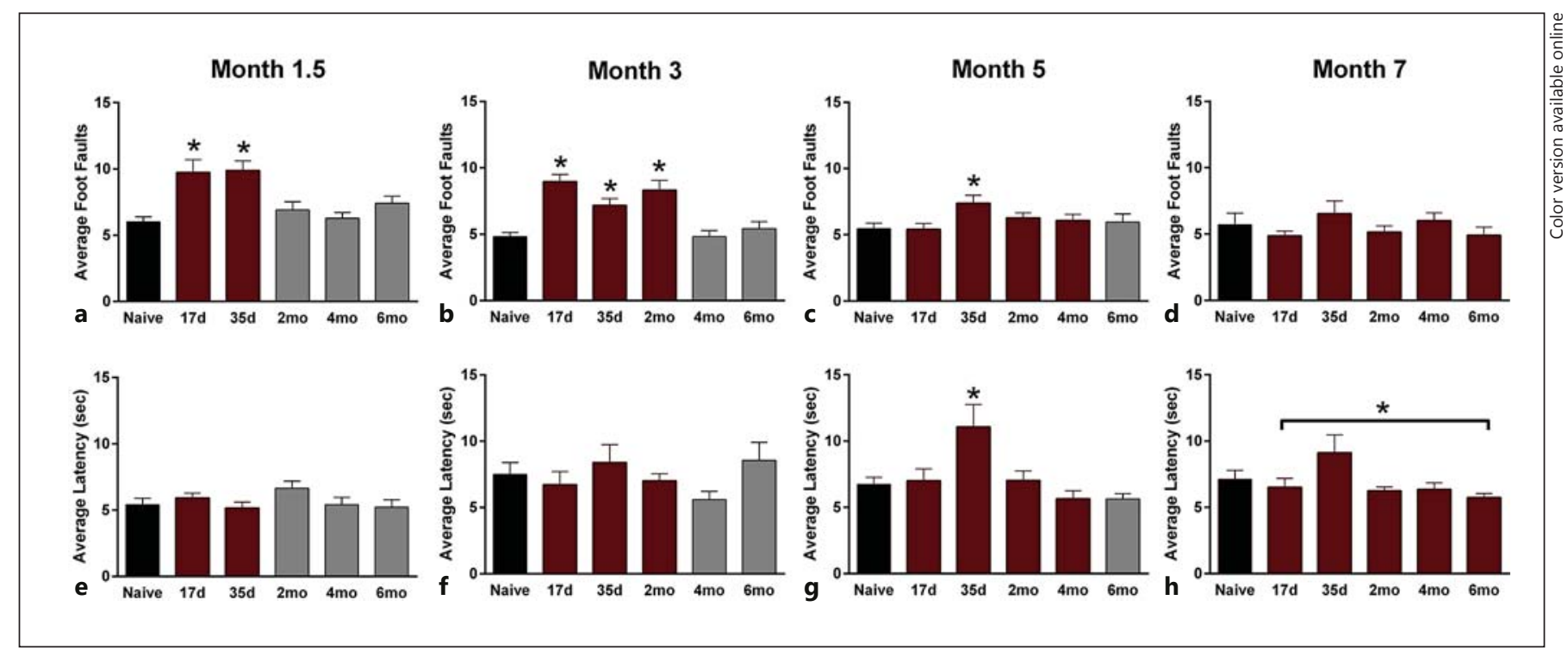

Fig. 3. Diffuse TBI resulted in vestibulomotor deficits as a function of age at injury. a-d Diffuse TBI resulted in a significant increase in average foot faults while crossing a $3-\mathrm{cm}$ wooden beam compared to naïve rats at $1.5,3$, and 5 months of age. $\mathbf{e}-\mathbf{h}$ There were no significant differences in latency to cross the beam between brain-injured rats and naïve rats at 1.5 and 3 months (e, f); however, there were overall injury effects on latency to cross the beam at 5 and 7 months $(\mathbf{g}, \mathbf{h})$. Maroon bars (color in online version

rats were given $3 \mathrm{~min}$ to explore the objects. Exploration of the object was defined as the nose being within $2 \mathrm{~cm}$ of the object. The animals were tracked using an overhead camera, and rat movement was analyzed using EthoVision XT 10 software. Time spent with the novel object was recorded as the duration of each visit to the object in the novel location.

\section{Forced Swim Task}

Depressive-like behavior was assessed using the forced swim task, as previously described $[31,32]$. The rats were placed into black plastic containers $(38 \times 30 \times 67 \mathrm{~cm}, \mathrm{~L} \times \mathrm{W} \times \mathrm{H})$ filled with water $\left(25^{\circ} \mathrm{C}\right)$ for $6 \mathrm{~min}$. The first minute was excluded from the analysis as an acclimation phase. The rats were recorded using an overhead camera. Videos were analyzed by an individual blinded to the treatment groups for time spent actively seeking escape (included clawing at edges, aggressively swimming, and diving) and number of escape attempts.

\section{Statistical Analysis}

Data are shown as means \pm SEM and analyzed using statistical software (GraphPad Prism 6). Differences among age-at-injury groups were determined by a one-way ANOVA followed by Dunnett's multiple comparison test compared to naïve rats. Body weight was analyzed using a two-way repeated-measures ANOVA. Outliers were eliminated using Grubbs' test for outliers. Statistical significance was assigned when $\mathrm{p}<0.05$.

Aging with TBI only) indicate groups that had received brain injuries at the time of behavioral testing (gray bars of groups yet to be brain injured shown for reference but not included in statistical analyses). Data are presented as means \pm SEM; naïve: $\mathrm{n}=12,17$ days: $\mathrm{n}=11,35$ days: $\mathrm{n}=12,2$ months: $\mathrm{n}=11,4$ months: $\mathrm{n}=12,6$ months: $\mathrm{n}=12$. $* \mathrm{p}<0.05$, indicates significant difference from naïve using Dunnett's multiple comparisons test.

\section{Results}

\section{Diffuse TBI Did Not Affect Body Weight}

Rats were weighed biweekly for the duration of the study to monitor how age at injury influenced early and extended recovery periods. There was an overall significant time effect $\left(\mathrm{F}_{10,760}=3,904, \mathrm{p}<0.0001\right.$; fig. 2$)$ as all groups gained weight as they aged. There was no significant effect of age at injury on weight $\left(F_{5,76}=0.9276\right.$, $\mathrm{p}=0.4680$; fig. 2).

\section{Diffuse TBI Resulted in Vestibulomotor Deficits as a}

Function of Age at Injury

Vestibulomotor function was assessed by the beam walk task as the latency to cross the beam and number of foot faults that occurred during the traverse. Diffuse TBI resulted in a significant increase in average foot faults while crossing $1 \mathrm{~m}$ of a 3 -cm wide wooden beam compared to the naïve group of rats at 1.5 months of age $\left(F_{2,32}=9.411, p=0.0006\right.$; fig. 3a $), 3$ months of age $\left(F_{3,42}\right.$ $=11.53, \mathrm{p}<0.0001$; fig. $3 \mathrm{~b})$, and 5 months of age $\left(\mathrm{F}_{4,52}=\right.$ 2.863, $\mathrm{p}=0.0322$; fig. $3 \mathrm{c}$ ). Further analysis using Dun- 


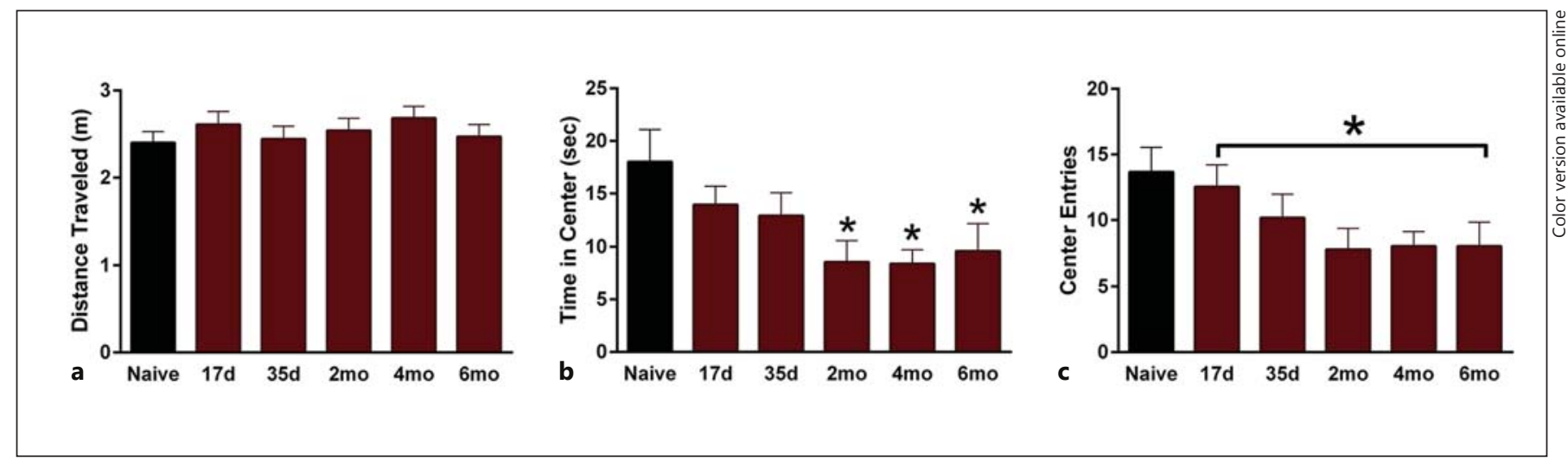

Fig. 4. Diffuse TBI resulted in age-at-injury-dependent anxiety at 8 months after injury. a There were no significant differences in the distance traveled during the open field task across all groups. However, there was an overall effect on total time spent in the center of the arena (b) and total entries into the center of the arena (c).
Data are presented as means \pm SEM; naïve: $\mathrm{n}=12,17$ days: $\mathrm{n}=11$, 35 days: $\mathrm{n}=12,2$ months: $\mathrm{n}=11,4$ months: $\mathrm{n}=12,6$ months: $\mathrm{n}=$ 12. ${ }^{*} \mathrm{p}<0.05$, indicates significant difference from naïve using Dunnett's multiple comparisons test.

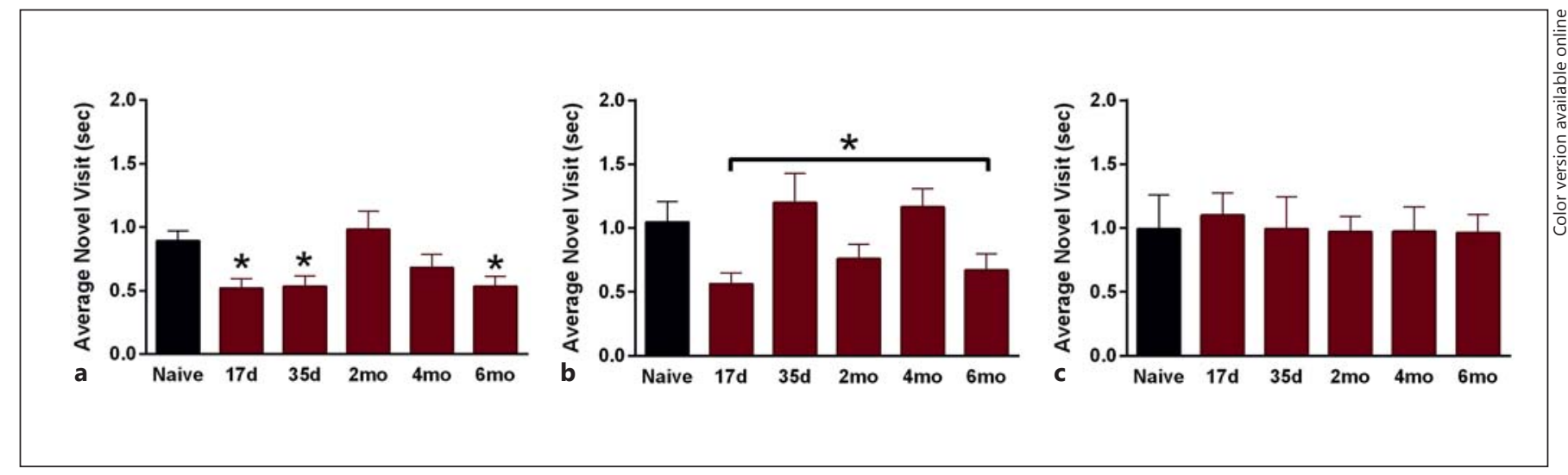

Fig. 5. Diffuse TBI resulted in age-at-injury-dependent spatial memory deficits. There was an overall effect on the average duration of visits to the novel location at 8 months (a) and 9 months of age (b). Rats injured at PND 17 and PND 35 had significantly shorter explorations of the object in the novel location compared to naïve rats. $\mathbf{c}$ There was no overall effect on duration of visits to the novel location at 10 months. Data are presented as means \pm SEM; naïve: $\mathrm{n}=12,17$ days: $\mathrm{n}=11,35$ days: $\mathrm{n}=12,2$ months: $\mathrm{n}=$ 11, 4 months: $\mathrm{n}=12,6$ months: $\mathrm{n}=12 .^{*} \mathrm{p}<0.05$, indicates significant difference from naïve using Dunnett's multiple comparisons test. nett's multiple comparisons test indicated all injured groups had significantly more foot faults compared to uninjured naïve rats at both 1.5 and 3 months, and rats injured at PND 35 had significantly more foot faults at 5 months. At 7 months of age, all groups performed similarly when measuring the number of foot faults $\left(\mathrm{F}_{5,62}=\right.$ $0.9126, p=0.4789$; fig. $3 \mathrm{~d}$ ). There were no significant differences in latency to cross the beam between brain-injured rats and naïve rats at 1.5 months $\left(\mathrm{F}_{2,32}=0.7878\right.$, $\mathrm{p}=0.4635$; fig. $3 \mathrm{e})$ and 3 months $\left(\mathrm{F}_{3,42}=0.5374, \mathrm{p}=\right.$ 0.6593 ; fig. 3f). However, there was an overall injury effect on latency to cross the beam at 5 months $\left(\mathrm{F}_{4,52}=4.405\right.$, $\mathrm{p}=0.0038$; fig. $3 \mathrm{~g}$ ), and Dunnett's multiple comparisons test indicated a significant increase in the latency of rats injured at PND 35 compared to naïve rats. Similarly, there was an overall injury effect on latency to cross the beam at 7 months $\left(F_{5,62}=2.618, p=0.0327\right.$; fig. $\left.3 \mathrm{~h}\right)$, with no specific group effects. 
Fig. 6. Diffuse TBI did not result in depressive-like behavior. There was no overall effect on the amount of time spent actively seeking escape (a) or the total amount of escape attempts (b) in the forced swim test. Data are presented as means \pm SEM; naïve: $\mathrm{n}=12,17$ days: $\mathrm{n}=11,35$ days: $\mathrm{n}=12,2$ months: $\mathrm{n}=11,4$ months: $\mathrm{n}=12,6$ months: $\mathrm{n}=12$.

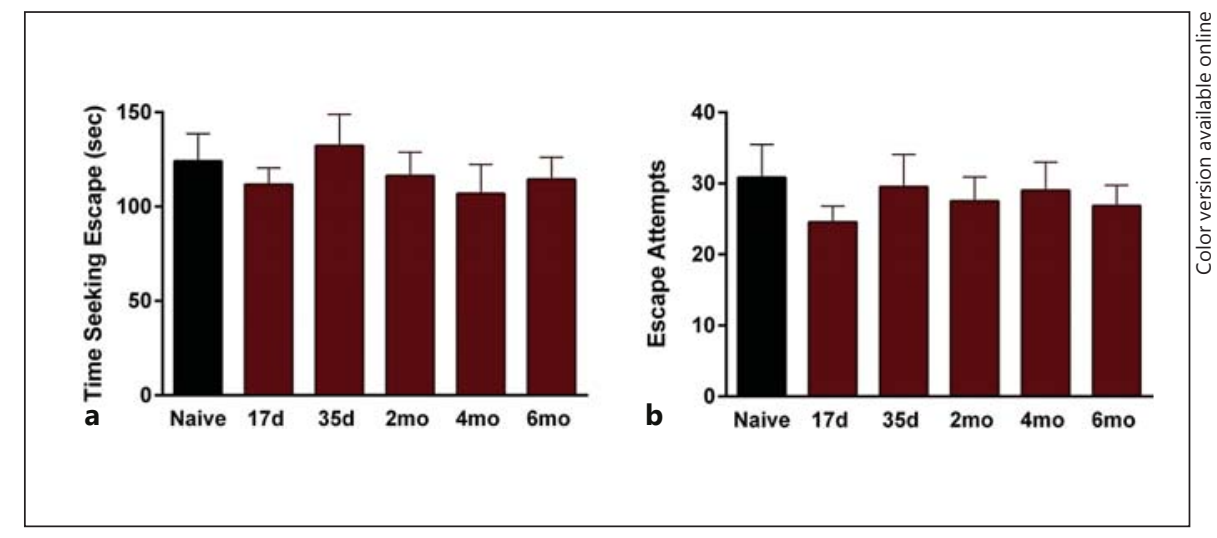

Diffuse TBI Resulted in Age-at-Injury-Dependent

Anxiety at 8 Months after Injury

Diffuse TBI resulted in age-at-injury-dependent anxiety at 8 months of age assessed by the open field task. There were no significant differences in the distance traveled during the task across all groups $\left(\mathrm{F}_{5,64}=0.6091, \mathrm{p}=\right.$ 0.6932; fig. 4a). However, there was an overall effect on total time spent in the center of the arena $\left(\mathrm{F}_{5,64}=2.879\right.$, $\mathrm{p}=0.0209$; fig. $4 \mathrm{~b}$ ). Dunnett's multiple comparisons test indicated that rats injured at 2, 4, and 6 months spent less time in the center of the arena, an anxiety-like behavior, compared to uninjured naïve rats. Overall, brain-injured rats had significantly fewer entries into the center of the arena compared to naïve rats $\left(\mathrm{F}_{5,64}=2.367, \mathrm{p}=0.0493\right.$; fig. 4c), with no specific effects.

\section{Diffuse TBI Resulted in Age-at-Injury-Dependent Spatial Memory Deficits}

Diffuse TBI led to deficits in spatial memory as assessed with a novel location task. There was an overall effect on the average duration of visits to the novel location at 8 months $\left(\mathrm{F}_{5,63}=4.334, \mathrm{p}=0.0019\right.$; fig. 5a $)$ and 9 months $\left(\mathrm{F}_{5,63}=\right.$ $3.176, p=0.0128$; fig. 5b). Dunnett's multiple comparisons tests indicated that rats injured at PND 17 and PND 35 had significantly fewer visits to the novel location compared to naïve rats. There was no overall effect on visits to the novel location at 10 months $\left(F_{5,63}=0.065, p=0.9970\right.$; fig. $\left.5 c\right)$.

\section{Diffuse TBI Did Not Result in Depressive-Like \\ Behavior}

Depressive-like behavior was assessed using the forced swim task. There was no overall effect on the amount of time spent actively trying to escape $\left(\mathrm{F}_{5,64}=0.5184, \mathrm{p}=\right.$ 0.7614 ; fig. 6a) or the total number of escape attempts $\left(\mathrm{F}_{5,64}=0.3124, \mathrm{p}=0.9038\right.$; fig. $\left.6 \mathrm{~b}\right)$.

\section{Discussion}

The chronic consequences of TBI impact cognitive and emotional health, with symptoms affecting somatic sensory systems, neurobehavioral and cognitive complaints, and neuropsychiatric issues related to emotional difficulties [33]. While TBI affects millions each year, the most recent data from the Center for Disease Control and Prevention (CDC) identifies the highest incidence rates for TBI among young children aged $0-4$ years and older adolescents aged 15-19 years, with adults aged over 75 also having a high incidence rate [33]. These data highlight the need for further research on brain injury during development and aging processes. In the debate of plasticity versus early vulnerability in children, controversy exists regarding whether the immature brain is at a maximal level of plasticity, and thus less susceptible to the impact of brain injury, or if the immature brain is uniquely sensitive to insult and TBI is detrimental to development $[3,13]$. Furthermore, little is known about the chronic interactions between injury and the aging brain. Here, we implemented a longitudinal study design to investigate the effects of age at injury on long-term functional outcome, including somatic, cognitive, and psychiatric measures. We found a single diffuse brain injury led to motor and cognitive deficits as a function of age, with rats injured during developmental stages equivalent to childhood and adolescence having significant motor and cognitive deficits compared to uninjured naïve rats. We also found anxiety-like behavior deficits in rats injured after the onset of adulthood but not in rats injured in earlier developmental phases. These data indicate differential windows of vulnerability to brain injury during development and aging. 
When brain injury was acquired during developmental stages of the rat equivalent to young childhood (PND 17) and adolescence (PND 35), vestibulomotor deficits were evident, as measured by foot faults while crossing a wooden beam compared to naïve rats. This finding is consistent with rats injured at PND 17 with a focal controlled cortical impact (CCI) model that had significantly more foot faults on a beam walk than uninjured rats at 1 , 7,14 , and 28 days after injury [34]. A similar study showed that PND 17 rats subjected to CCI had significantly more foot faults on a foot fault task measuring motor coordination and proprioception at 3 and 5 months after injury compared to uninjured shams [35]. Our data and these studies indicate an early vulnerability for vestibulomotor dysfunction in rats injured at PND 17 and PND 35 which persists up to 4 months after injury. Our data extend these findings to 6 months after injury, by which time these impairments were resolved.

In the current study, rat brains injured during these critical stages of development also presented with cognitive problems, as measured by working spatial memory deficits in the novel object location task. The brains of children are rapidly developing, and neurobehavioral skills are immature. Acquiring a TBI can disrupt this essential development. Clinical data from pediatric TBI survivors suggest deficits in cognition, executive function, and memory $[36,37]$. For example, children who suffer from TBI before the age of 2 years were found to be at a high risk for developing cognitive deficits and had persisting cognitive delays up to a year after brain injury [38]. Furthermore, our data affirm existing experimental animal data showing impaired recovery from diffuse brain injury in younger animals [39]. Rats subjected to a diffuse TBI using a weight drop model of brain injury at PND 3 and PND 7 demonstrated a rapid age-dependent decline in neurological outcomes [39]. PND 17 rats subjected to diffuse impact acceleration had significantly longer latencies to find the hidden platform during the Morris water maze task compared to shams at 1,2 , and 3 months after injury [40]. These data, along with our results, suggest vulnerability of the immature brain to diffuse TBI and support the early vulnerability perspective of juvenile TBI rather than increased plasticity affording greater capacity for recovery in the immature brain.

While treatment and rehabilitation efforts for TBI primarily focus on sensory, motor, and cognitive symptoms, neuropsychiatric disorders are responsible for emotional and behavioral difficulties contributing to an impaired quality of life [41]. Psychiatric conditions are significantly more frequent in TBI survivors compared to healthy controls $[42,43]$, probably arising from neurobiological and pathophysiological changes initiated by TBI [44-46]. Anxiety disorders following TBI can be caused by disruption of neurotransmitters, disruption of the neuroendocrine system, structural responses to physical injury, and the incomplete management of postconcussive symptoms [47]. In the current study, we found that rats injured during adulthood developed anxietylike behavior assessed by the open field task, but rats injured in childhood or adolescence did not develop a chronic anxiety compared to uninjured naïve rats. Similar to our findings, a study investigating experimental juvenile TBI using focal CCI found that rats injured at PND 17 did not develop anxiety-like affective behaviors as assessed with the zero maze at 3,5, or 6 months after injury compared to uninjured shams [35]. Clinical data support this finding in that patients who were over the age of 50 when they acquired a TBI did not present cognitive deficits but reported significant anxiety [9]. There is controversy in the clinical data whether anxiety following TBI is most profound shortly following TBI or whether it develops years after injury $[47,48]$. The prevalence of anxiety following adult TBI has been described as being most prevalent 2-5 years after injury [48], but contrarily has also been described to be most profound shortly following injury [47]. The longitudinal study design used here evaluated anxiety at 8 months of age, which represents various times after injury for each injured group of rats. It is possible that the rats injured in adulthood had significant anxiety-like behavior compared to uninjured naïve rats, because the test was administered closer to their time of injury compared to rats injured in childhood (PND 17) and adolescence (PND 35). A recent retrospective clinical study reported no significant correlation between age at injury and psychological outcome parameters [49]. Few studies have evaluated the development of anxiety disorders after TBI, and further studies are necessary to understand the mechanisms of psychopathology among TBI survivors.

The most common psychiatric complaint of patients following mild TBI is depression [50, 51]. Clinical hallmarks of depression include anhedonia, loss of interest in pleasure derived from typically enjoyable activities, and behavioral despair. In rodents, behavioral despair is characterized by a significant decline in an animal's effort to avoid or escape aversive situations [31, 41]. In the current study, we did not find depressive-like behavior in braininjured rats compared to uninjured naïve rats regardless of age at injury as assessed with the forced swim task. 
Similarly, using lateral fluid percussion brain injury, no differences in swimming activity were found between brain-injured rats and uninjured controls at 6 months after injury [52]. However, rats subjected to a focal brain injury by CCI exhibited less swimming behavior compared to uninjured control rats at 8 weeks after injury, suggesting depression-like symptoms in the brain-injured rats [53]. There are limitations in using rodents because it is impossible to fully replicate emotional reactions and expectations of individuals along with their social interactions [41]. Furthermore, inconsistencies in the development of depression in rats subjected to experimental TBI could be explained by differences in injury model and location of injury, as well as varying testing procedures across laboratories.

Clinical data support that a history of TBI combined with brain changes associated with normal aging can advance cognitive decline in older adults $[54,55]$. Patients in a TBI group made significantly more errors on a test measuring executive functioning compared to an uninjured control group, further supporting that TBI puts older adults at a higher risk for exacerbated age-related cognitive decline [56]. Epidemiological studies also identify TBI as a risk factor for the development of dementia and Alzheimer's disease [57]. The current study did not assess cognitive decline past the age of 10 months, when laboratory rats can live to 24 or more months of age. As average life spans continue to increase, further attention is directed toward both aging with brain injury and acquiring a brain injury at an older age. Future studies are necessary to measure cognitive impairment in rats that are aged beyond 10 months to further elucidate the role of TBI and aging on cognitive decline.

This study incorporated several novel features. In order to induce diffuse brain injury in rodents of different ages, three different trephine diameters were used, proportional to the area of the rat skull. At each age, the area of the craniotomy was approximately $50 \%$ of the distance between bregma and lambda skull sutures. This normalization was intended to have a similar impact area for each age-at-injury group. Further, injury pressure levels were empirically derived to achieve 6-10 min of suppression of the righting reflex across age groups as an equivalent neurological response to injury. The study design was further strengthened by having a single cohort of animals exposed to identical housing conditions and seasonal effects. The compromise did not include uninjured sham animals, which would have made behavioral testing unmanageable. However, we view the brain injury as including the surgical preparation.
While having a single cohort of animals strengthened the study design, the practical aspects of working with a large cohort of animals created limitations. The battery of evaluated behaviors necessitated a singular approach to be feasible within a narrow experimental testing time window. As such, the current study design used a single test of cognitive function, anxiety-like, and depressivelike behaviors. Future studies are necessary to more fully elucidate the role of diffuse brain injury on cognitive and affective age-dependent deficits. Future experiments investigating these lasting impairments across each specific age can include the Morris water maze and novel object recognition to test cognition, and the elevated plus maze and sucrose preference test to evaluate affective functions.

\section{Conclusion}

Overall, the current study supports the conclusion that TBI can have a negative impact during discrete stages of development and aging. Clinical management must continue to consider the developmental stage of the child at the time of TBI when treating and predicting outcomes. Previous studies focus primarily on focal TBI in immature rats; this study is one of the first of its kind to use a well-established model of diffuse brain injury to investigate effects of age at injury on behavioral outcome. The authors recognize that histopathological outcomes can influence the development of behavioral deficits, and while it was beyond the scope of this project, future studies are underway to investigate pathological outcomes as a function of age at injury.

\section{Acknowledgments}

The authors would like to thank Mr. Tony Romero and Mr. Mahir Qureshi for their technical assistance. The research reported in this paper was supported primarily by the Arizona Alzheimer's Consortium with matching funds from the University of Arizona, College of Medicine - Phoenix and Barrow Neurological Institute at Phoenix Children's Hospital. Partial support was provided by the National Institute of Neurological Disorders and Stroke of the National Institutes of Health under award number R01 NS-065052. Dr. Rachel K. Rowe was supported by the Bisgrove Scholar award funded by Science Foundation Arizona. Jordan L. Harrison was supported, in part, by the Bruce and Diane Halle Foundation and NIH fellowship F31-NS090921. L. Matthew Law was supported by the Bruce and Diane Halle Foundation. 


\section{References}

$>1$ Werner C, Engelhard K: Pathophysiology of traumatic brain injury. Br J Anaesth 2007;99: 4-9.

2 Babikian T, et al: Chronic aspects of pediatric traumatic brain injury: review of the literature. J Neurotrauma 2015;32:1849-1860.

$>3$ Giza CC, Prins ML: Is being plastic fantastic? Mechanisms of altered plasticity after developmental traumatic brain injury. Dev Neurosci 2006;28:364-379.

4 Schneider GE: Is it really better to have your brain lesion early - revision of the 'Kennard principle’. Neuropsychologia 1979;17:557583.

5 Kennard MA: Age and other factors in motor recovery from precentral lesions in monkeys. Am J Physiol 1936;115:138-146.

$\checkmark 6$ Crowe LM, et al: Timing of traumatic brain injury in childhood and intellectual outcome. J Pediatr Psychol 2012;37:745-754.

7 Ballantyne AO, et al: Plasticity in the developing brain: intellectual, language and academic functions in children with ischaemic perinatal stroke. Brain 2008;131:2975-2985.

$>8$ Thomas S, et al: Influences of secondary injury following traumatic brain injury in developing versus adult rats. Acta Neurochir Suppl 2000;76:397-399.

$>9$ Goldstein FC, Levin HS: Cognitive outcome after mild and moderate traumatic brain injury in older adults. J Clin Exp Neuropsychol 2001;23:739-753.

$\checkmark 10$ Scholten AC, et al: Prevalence and risk factors of anxiety and depressive disorders following traumatic brain injury: a systematic review. J Neurotrauma 2016, Epub ahead of print.

$>11$ Sun D, et al: Aging- and injury-related differential apoptotic response in the dentate gyrus of the hippocampus in rats following brain trauma. Front Aging Neurosci 2013;5:95.

12 Gaastra B, et al: The ageing population is neglected in research studies of traumatic brain injury. Br J Neurosurg 2016;30:221-226.

$>13$ Anderson V, et al: Childhood brain insult: can age at insult help us predict outcome? Brain 2009; 132:45-56.

14 Donders J, Warschausky S: Neurobehavioral outcomes after early versus late childhood traumatic brain injury. J Head Trauma Rehabil 2007;22:296-302.

$>15$ Goodus MT, et al: Neural stem cells in the immature, but not the mature, subventricular zone respond robustly to traumatic brain injury. Dev Neurosci 2015;37:29-42.

16 Lifshitz J: Fluid percussion injury model; in Chen J, et al (eds): Animal Models of Acute Neurological Injuries. Totowa, Humana Press, 2007, pp 369-384.

-17 McNamara KC, Lisembee AM, Lifshitz J: The whisker nuisance task identifies a late-onset, persistent sensory sensitivity in diffuse braininjured rats. J Neurotrauma 2010;27:695-706.
18 Ziebell JM, et al: Rod microglia: elongation, alignment, and coupling to form trains across the somatosensory cortex after experimental diffuse brain injury. J Neuroinflammation 2012;9:247.

19 Cao T, et al: Morphological and genetic activation of microglia after diffuse traumatic brain injury in the rat. Neuroscience 2012; 225:65-75.

20 Lifshitz J: Fluid percussion injury; in Chen J, $\mathrm{Xu}$ X-M, Zhang J (eds): Animal Models of Acute Neurological Injuries. Totowa, Humana Press, 2008.

21 Thomas TC, et al: Hypersensitive glutamate signaling correlates with the development of late-onset behavioral morbidity in diffuse brain-injured circuitry. J Neurotrauma 2012; 29:187-200.

22 Prins ML, et al: Fluid percussion brain injury in the developing and adult rat: a comparative study of mortality, morphology, intracranial pressure and mean arterial blood pressure. Brain Res Dev Brain Res 1996;95:272-282.

-23 Rowe RK, et al: Recovery of neurological function despite immediate sleep disruption following diffuse brain injury in the mouse: clinical relevance to medically untreated concussion. Sleep 2014;37:743-752.

24 Rowe RK, et al: Diffuse brain injury induces acute post-traumatic sleep. PLoS One 2014;9: e82507.

25 Rowe RK, et al: Diffuse brain injury does not affect chronic sleep patterns in the mouse. Brain Inj 2014;28:504-510.

26 Harrison JL, et al: Resolvins AT-D1 and E1 differentially impact functional outcome, post-traumatic sleep, and microglial activation following diffuse brain injury in the mouse. Brain Behav Immun 2015;47:131140.

27 Soltani Z, et al: Is genistein neuroprotective in traumatic brain injury? Physiol Behav 2015; 152:26-31.

28 Feeney DM, Gonzalez A, Law WA: Amphetamine, haloperidol, and experience interact to affect rate of recovery after motor cortex injury. Science 1982;217:855-857.

29 Nagai T, et al: D-Serine ameliorates neonatal PolyI:C treatment-induced emotional and cognitive impairments in adult mice. J Pharmacol Sci 2012;120:213-227.

30 Zhang RS, et al: Endomorphin-1 attenuates A $\beta 42$ induced impairment of novel object and object location recognition tasks in mice. Brain Res 2015;1629:210-220.

31 Porsolt RD, et al: Behavioural despair in rats: a new model sensitive to antidepressant treatments. Eur J Pharmacol 1978;47:379-391.

32 Porsolt RD: Animal model of depression. Biomedicine 1979;30:139-140.
33 Cole WR, Bailie JM: Neurocognitive and psychiatric symptoms following mild traumatic brain injury; in Laskowitz D, Grant G (eds): Translational Research in Traumatic Brain Injury. Boca Raton, CRC Press, 2016.

34 Russell KL, et al: Sensorimotor behavioral tests for use in a juvenile rat model of traumatic brain injury: assessment of sex differences. J Neurosci Methods 2011;199:214-222.

35 Kamper JE, et al: Juvenile traumatic brain injury evolves into a chronic brain disorder: behavioral and histological changes over 6 months. Exp Neurol 2013;250:8-19.

36 Anderson V, et al: Intellectual outcome from preschool traumatic brain injury: a 5-year prospective, longitudinal study. Pediatrics 2009; 124:e1064-e1071.

\$3 Jaffe KM, et al: Severity of pediatric traumatic brain injury and neurobehavioral recovery at one year - a cohort study. Arch Phys Med Rehabil 1993;74:587-595.

38 Keenan HT, et al: Neurodevelopmental consequences of early traumatic brain injury in 3-year-old children. Pediatrics 2007;119: e616-e623.

39 Bittigau P, et al: Apoptotic neurodegeneration following trauma is markedly enhanced in the immature brain. Ann Neurol 1999;45: 724-735.

40 Adelson PD, Dixon CE, Kochanek PM: Longterm dysfunction following diffuse traumatic brain injury in the immature rat. J Neurotrauma 2000;17:273-282.

41 Malkesman O, et al: Traumatic brain injury - modeling neuropsychiatric symptoms in rodents. Front Neurol 2013;4:157.

42 McAllister TW, Green RL: Traumatic brain injury: a model of acquired psychiatric illness? Semin Clin Neuropsychiatry 1998;3: 158-159.

43 Mallya S, et al: The manifestation of anxiety disorders after traumatic brain injury: a review. J Neurotrauma 2015;32:411-421.

44 Broshek DK, De Marco AP, Freeman JR: A review of post-concussion syndrome and psychological factors associated with concussion. Brain Inj 2015;29:228-237.

45 Reger ML, et al: Concussive brain injury enhances fear learning and excitatory processes in the amygdala. Biol Psychiatry 2012;71:335343.

46 Hudak A, et al: Brain morphometry changes and depressive symptoms after traumatic brain injury. Psychiatry Res 2011;191:160165.

47 Fann JR, Jakupcak M: Management of Adults with Traumatic Brain Injury. Arlington, American Psychiatric Publishing, 2013.

48 Osborn AJ, Mathias JL, Fairweather-Schmidt AK: Prevalence of anxiety following adult traumatic brain injury: a meta-analysis comparing measures, samples and postinjury intervals. Neuropsychology 2016;30:247-261. 
49 Andruszkow H, et al: Physical and psychological long-term outcome after traumatic brain injury in children and adult patients. Health Qual Life Outcomes 2014;12:26.

50 Riggio S, Wong M: Neurobehavioral sequelae of traumatic brain injury. Mt Sinai J Med 2009;76:163-172.

-51 Silver JM, McAllister TW, Arciniegas DB: Depression and cognitive complaints following mild traumatic brain injury. Am J Psychiatry 2009;166:653-661.
52 Jones NC, et al: Experimental traumatic brain injury induces a pervasive hyperanxious phenotype in rats. J Neurotrauma 2008;25:13671374.

53 Taylor AN, et al: Lasting neuroendocrine-immune effects of traumatic brain injury in rats. J Neurotrauma 2006;23:1802-1813.

54 Moretti L, et al: Cognitive decline in older adults with a history of traumatic brain injury. Lancet Neurol 2012;11:1103-1112.
55 Plassman BL, et al: Documented head injury in early adulthood and risk of Alzheimer's disease and other dementias. Neurology 2000; 55:1158-1166.

56 Ozen LJ, et al: Evidence of cognitive decline in older adults after remote traumatic brain injury: an exploratory study. Neuropsychol Dev Cogn B Aging Neuropsychol Cogn 2015;22: 517-533.

57 Jellinger KA: Head injury and dementia. Curr Opin Neurol 2004;17:719-723. 